\title{
Sulfonated Non-Saccharide Heparin Mimetics are Potent and Noncompetitive Inhibitors of Human Neutrophil Elastase
}

\author{
Rami A. Al-Horani, ${ }^{1}$ Kholoud F. Aliter, ${ }^{2}$ Srabani Kar, ${ }^{1}$ Madhusoodanan Mottamal ${ }^{3}$ \\ ${ }^{1}$ Division of Basic Pharmaceutical Sciences, College of Pharmacy, Xavier University of \\ Louisiana, New Orleans LA 70125, ralhoran@xula.edu \\ ${ }^{2}$ Department of Chemistry, School of STEM, Dillard University, \\ NewOrleans LA 70122, kal-horani@dillard.edu \\ ${ }^{3}$ Department of Chemistry, Xavier University of Louisiana, New Orleans, LA 70125, USA \\ mmottama@xula.edu
}

\section{Testing inhibitor 3 effect on hydrolysis of FIX by HNE.}

\section{Experiment.}

FIX $(4.0 \mu \mathrm{M})$ was incubated at $37^{\circ} \mathrm{C}$ with HNE $(400 \mathrm{nM})$ in pH 7.4 HEPES buffer for $30 \mathrm{~min}$. Aliquot was withdrawn from the reaction mixture to which an equal volume of SDS solution containing dithiothreitol was added, and the resulting mixture was heated for 2 minutes at $90^{\circ} \mathrm{C}$ for SDS-PAGE. The mixture was then electrophoresed on a 10\% SDS-PAGE. Protein bands were visualized by staining with silver stain. The experiment was done in the absence or presence 5 or $20 \mu \mathrm{M}$ inhibitor 3

\section{Results.}

We did SDS-PAGE analysis as previously published. ${ }^{1}$ We noticed that incubating HNE with FIX resulted in three small $(30,20,17 \mathrm{kD}$, lane 4 below), detected fragments on SDS-PAGE, as previously reported, ${ }^{1}$ however when we re-performed the test with $5 \mu \mathrm{M}$ (and $20 \mu \mathrm{M}$ ) inhibitor 3, we detected FIX (not hydrolyzed), and two fragments (lane 5) one with a relatively high molecular weight and another small fragment that is fundamentally different from what was found in the absence of inhibitor 3. While it is difficult now to fully explain what the effect of the inhibitor on the hydrolysis of FIX by HNE was, yet clearly there is an effect in which less hydrolysis of FIX took place and two new fragments, one of which has a molecular weight close to the parent protein i.e. FIX. This suggests that inhibitor $\mathbf{3}$ does indeed affect the catalytic activity of HNE, even using some of its physiological substrates. 


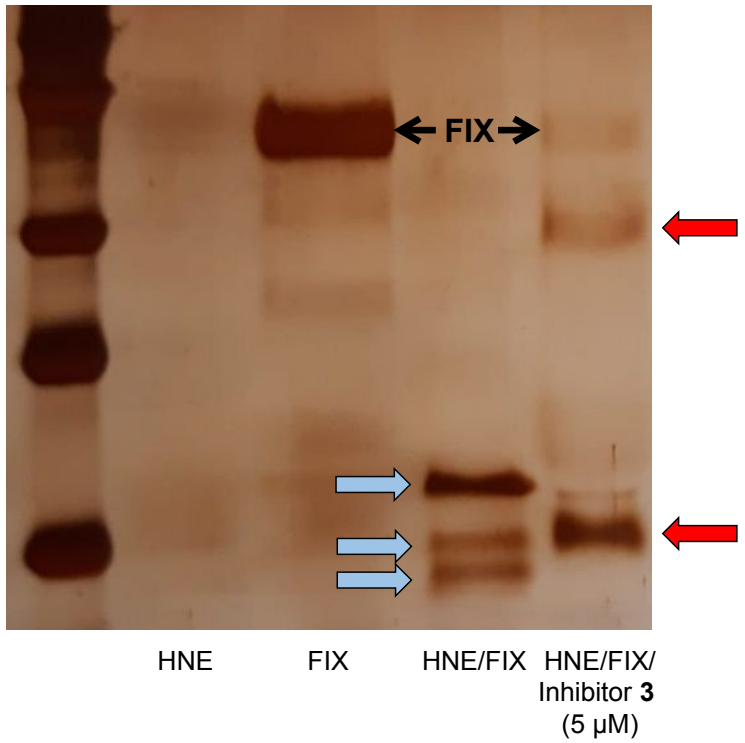

Figure S1. Effect of HNE on FIX in the presence and absence of inhibitor 3 using reducing SDS-PAGE.

\section{Reference.}

(1) Samis, J.A.; Kam, E.; Nesheim, M.E.; Giles, A.R. Neutrophil elastase cleavage of human factor IX generates an activated factor IX-like product devoid of coagulant function. Blood. 1998, 92, 1287-96. 